\title{
Utility Maximization in Incomplete Markets with Random Endowment
}

\author{
Jakša Cvitanić * \\ Department of Mathematics \\ USC, 1042 W 36 Pl, DRB 155 \\ Los Angeles, CA 90089-1113 \\ cvitanic@math.usc.edu \\ Walter Schachermayer ${ }^{\dagger}$ \\ Department of Statistics, Probability Theory and Actuarial Mathematics \\ Vienna University of Technology \\ Wiedner Hauptstrasse 8-10, A-1040 Wien \\ wschach@fam.tuwien.ac.at \\ Hui Wang \\ Department of Statistics \\ Columbia University \\ New York, NY 10027 \\ wanghui@stat. columbia.edu
}

December 20, 2001

\begin{abstract}
This paper solves a long-standing open problem in mathematical finance: to find a solution to the problem of maximizing utility from terminal wealth of an agent with a random endowment process, in the general, semimartingale model for incomplete markets, and to characterize it via the associated dual problem. We show that this is indeed possible if the dual problem and its domain are carefully defined. More
\end{abstract}

${ }^{*}$ Research supported in part by the NSF Grant DMS-97-32810; on leave from Columbia University.

${ }^{\dagger}$ Support by the Austrian Science Foundation (FWF) under grant SFB\#010 and by the Austrian National Bank under grant 'Jubilaumsfondprojekt Number 7049' is greatfully acknowledged. 
precisely, we show that the optimal terminal wealth is equal to the inverse of marginal utility evaluated at the solution to the dual problem, which is in the form of the regular part of an element of $\left(\mathbf{L}^{\infty}\right)^{*}$ (the dual space of $\mathbf{L}^{\infty}$ ).

Key words: utility maximization, incomplete markets, random endowment, duality.

JEL classification: G11, G12; C61

AMS 1991 subject classifications: Primary 90A09, 90A10; secondary 90C26.

\section{Introduction}

The problem of utility maximization in incomplete markets is relatively new - it was solved in Ito-processes models of financial markets by Karatzas, Lehoczky, Shreve and Xu (1991) (henceforth KLSX[91]), using the powerful convex duality/martingale approach, which enabled the authors to deal with models which are not necessarily Markovian (for a more detailed history of the problem see Kramkov and Schachermayer (1999), henceforth KS[99]). The approach has recently been generalized to semimartingale models and under weaker conditions on the utility function by KS[99]. One of the main innovations of the latter article, that made the approach work in this general context, was the extension of the domain of the dual problem: it is defined through a family of random variables $Y(T)$ (here $T$ denotes the terminal time) associated with nonnegative processes $Y(\cdot)$ which are such that, for any admissible wealth processs $X(\cdot)$, the product process $X(\cdot) Y(\cdot)$ is a supermartingale, and not necessarily a local martingale as in KLSX[91]. In both KLSX[91] and KS[99], the agent was endowed with an initial capital $x>0$, and received no endowment after the initial time $t=0$. Attempts to extend the KLSX[91] approach to an agent who receives a random endowment process have failed (if the endowment process cannot be replicated in the market). Nevertheless, solutions have been found by attacking directly the primal problem in special cases: in Markovian models by Duffie, Fleming, Soner and Zariphopoulou (1997), and in more general models in Cuoco (1997). A dual problem approach in a particular Brownian model has been worked out under the constraint $X(\cdot) \geq 0$ by El Karoui and Jeanblanc (1998). This constraint is somewhat stringent in models with endowment process, since it precludes borrowing against future income. 
In this paper we solve in great generality the problem of maximizing expected utility $E[U(X(T))]$ of terminal wealth, for an agent whose income is represented as an arbitrary bounded and adapted endowment process $e(\cdot)$. This is done in the general semimartingale incomplete model, under the same minimal conditions on the utility function $U$ as in $\mathrm{KS}$ [99], and using a similar duality approach. The main difference, and the reason why we are able to do it, is that we extend the dual domain even further - it is no longer contained in the space $\mathbf{L}^{1}$, but $\left(\mathbf{L}^{\infty}\right)^{*}$, the dual space of $\mathbf{L}^{\infty}$. In the language of control theory, we are "relaxing" the set of controls over which we do the optimization in the dual problem. The solution $\hat{Q}$ is then found in this set, and the optimal terminal wealth is shown to be equal to the inverse of marginal utility evaluated at the regular part of $\hat{Q}$. It should be mentioned that this approach was already implicitly present in $\mathrm{KS}[99]$ : in that paper the domain of the dual problem is associated with processes $Y(t)$ which, in our context, correspond to the processes given by the Radon-Nikodym densities of the regular part of the restriction of elements $Q \in\left(\mathbf{L}^{\infty}\right)^{*}$ to $\mathcal{F}_{t}$, the $\sigma$-algebra generated by the information up to time $t$. It was shown in that article that the optimal $\hat{Y}(\cdot)$ is not necessarily a martingale, hence the corresponding $\hat{Q}$ is not necessarily contained in $\mathbf{L}^{1}$. It was not important for the analysis of KS[99] to observe where "the singular mass of $\hat{Q}$ has disappeared to". In the present paper this becomes very important, since the "disappeared mass" does not actually vanish, but acts on the accumulated random endowment, and can be located in $\left(\mathbf{L}^{\infty}\right)^{*}$.

We introduce the model and the primal problem in Section 1, and define the dual problem in Section 2. We solve it and make the connection to the primal problem in Section 3. Finally, in the Appendix we recall some results on properties of $\left(\mathbf{L}^{\infty}\right)^{*}$ needed in the paper.

\section{The Market Model}

We consider a model of a security market which consists of $d+1$ assets, one bond (or bank account) and $d$ stocks. Without loss of generality, we assume that the bond price is constant (we can always choose the bond as the numéraire otherwise). The stock-price process $S=\left(S^{i}\right)_{1 \leq i \leq d}$ is assumed to be a semimartingale on a filtered probability space $\left(\Omega, \mathcal{F},\left(\mathcal{F}_{t}\right)_{0 \leq t \leq T}, \mathbf{P}\right)$. Here $T$ is the finite time-horizon, but our results can also be extended to an infinite time-horizon.

A portfolio $\Pi$ is defined as a pair $(x, H)$, where the constant $x \in \mathbb{R}$ is the initial wealth and $H=\left(H^{i}\right)_{1 \leq i \leq d}$ is a predictable $S$-integrable process 
specifying the number of shares of each asset held in the portfolio. We also assume that the agent receives an exogenous endowment (income), with its cumulative process denoted by $e=\left(e_{t}\right)_{0 \leq t \leq T}, e_{0}=0$, assumed bounded and adapted, with $\rho \triangleq\left\|e_{T}\right\|_{\infty}<\infty$. The corresponding value process $A=\left(A_{t}\right)_{0 \leq t \leq T}$ is then given by

$$
A_{t}=x+(H \cdot S)_{t}+e_{t}, \quad 0 \leq t \leq T .
$$

Here $(H \cdot S)=\int_{0}^{\cdot} H d S$ denotes the stochastic integral with respect to $S$. Note that $e(\cdot)$ can take negative values, in which case it is interpreted as the mandatory consumption (mandatory outflow of funds). It should also be noted that for the problem of maximizing expected utility from terminal wealth $A_{T}$ that we consider here, only the final value $e_{T}$ matters. This is not the case when maximizing expected utility from consumption, a problem that we plan to consider in future research.

A portfolio $\Pi$ is called admissible if the process $(H \cdot S)$ is uniformly bounded from below by some constant. Let $\mathcal{C}_{0}$ be the convex cone of random variables dominated by admissible stochastic integrals, i.e.

$$
\mathcal{C}_{0} \triangleq\left\{X \mid X \leq(H \cdot S)_{T} \quad \text { for some admissible portfolio } H\right\}
$$

and $\mathcal{C} \triangleq \mathcal{C}_{0} \cap \mathbf{L}^{\infty}$, the intersection with space $\mathbf{L}^{\infty}$

Suppose that the agent also has a utility function $U:(0, \infty) \longrightarrow \mathbb{R}$ for wealth, which is strictly concave, strictly increasing, continuously differentiable and satisfies the Inada conditions

$$
U^{\prime}(0+)=\lim _{x \rightarrow 0} U^{\prime}(x)=\infty, \quad U^{\prime}(\infty)=\lim _{x \rightarrow \infty} U^{\prime}(x)=0 .
$$

Our primal problem is to maximize the expected utility from terminal wealth with value function

$$
u(x)=\max _{X \in \mathcal{C}_{0}} \mathrm{E}\left[U\left(x+X+e_{T}\right)\right] .
$$

Without loss of generality, we may assume $U(\infty)>0$. Define also $U(x)=$ $-\infty$ whenever $x \leq 0$.

We adopt the definition of an equivalent local martingale measure from $\mathrm{KS}[99]$.

Definition 2.1 A probability measure $\mathbf{Q} \sim \mathbf{P}$ is called an equivalent local martingale measure if for any $H$ admissible, $(H \cdot S)$ is a local martingale under $\mathbf{Q}$. 
Throughout the paper we shall assume the following conditions.

Assumption 1 The family of equivalent local martingale measures $\mathcal{M}$ is not empty.

Assumption 2 The utility function $U(x)$ has asymptotic elasticity strictly less than 1, i.e.

$$
A E(U) \triangleq \limsup _{x \rightarrow \infty} \frac{x U^{\prime}(x)}{U(x)}<1 .
$$

Assumption $3|u(x)|<\infty$ holds for some $x>\rho=\left\|e_{T}\right\|_{\infty}$.

Remark 2.1 Detailed discussions on Definition 2.1 and the intimate relationship between Assumption 1 and the absence of arbitrage opportunities are available in KS[99], DS[94] and DS[98] (for the case of general process $S$ which fails to be locally bounded).

Remark 2.2 It is shown in KS[99] that Assumption 2 is basically both necessary and sufficient to get existence and nice properties of the solution to the primal problem.

Remark 2.3 The concavity of $u(x)$ and Assumption 3 easily imply that $u(x)<\infty$ for all $x \in \mathrm{R}$.

\section{The Dual Problem}

Let us denote by $V:(0, \infty) \longrightarrow$ R the conjugate function of utility $U(x)$, i.e.,

$$
V(y) \triangleq \sup _{x>0}[U(x)-x y]=U(I(y))-y I(y) .
$$

Here $I:(0, \infty) \longrightarrow(0, \infty)$ is the continuous, strictly decreasing inverse function of the derivative of $U(x)$. It is well known that $V(y)$ is continuously differentiable, strictly decreasing, strictly convex and satisfies

$$
V(0)=U(\infty), \quad V(\infty)=U(0), \quad \text { and } \quad V^{\prime}=-I .
$$

The function $V(y)$ is the Legendre-transform of the function $-U(-x)$, which has been proved very useful in solving utility maximization problems, especially in non-Markovian cases (for early works on duality in stochastic optimal control see Bismut [73], and Pliska [86], for the first application to finance). In this paper, we extend the usual dual domain (a subset of $\mathbf{L}^{1}$ ) to 
$\left(\mathbf{L}^{\infty}\right)^{*}$, the dual space of $\mathbf{L}^{\infty}$. It is well known that $\mathbf{L}^{1}$ is strictly contained in $\left(\mathbf{L}^{\infty}\right)^{*}$; see Dunford and Schwartz (1967) or Appendix A for more details about space $\left(\mathbf{L}^{\infty}\right)^{*}$.

Define the following subset of $\left(\mathbf{L}^{\infty}\right)^{*}$, which is equipped with the weakstar topology:

$$
\mathcal{D} \triangleq\left\{Q \in\left(\mathbf{L}^{\infty}\right)^{*} \quad \mid \quad\|Q\|=1 \text { and }\langle Q, X\rangle \leq 0 \text { for all } X \in \mathcal{C}\right\},
$$

and $\mathcal{D}^{r} \triangleq \mathcal{D} \cap \mathbf{L}^{1}$ (where $r$ stands for "regular"). Note that $\mathcal{D} \subseteq\left(\mathbf{L}^{\infty}\right)_{+}^{*}$ (hence $\mathcal{D}^{r} \subseteq \mathbf{L}_{+}^{1}$ ) since $-\mathbf{L}_{+}^{\infty} \subseteq \mathcal{C}$. Moreover, $\mathcal{D}$ is clearly convex and weakstar compact (by Alaoglu's Theorem). For any $Q \in\left(\mathbf{L}^{\infty}\right)_{+}^{*}$, we have the unique decomposition $Q=Q^{r}+Q^{s}$. Here $Q^{r}$ and $Q^{s}$ are defined on the $\sigma$-algebra $\mathcal{F}$ modulo the nullsets; on this abstract $\sigma$-algebra $Q^{r}$ is countably additive and absolutely continuous while $Q^{s}$ is purely finitely additive.

For any $Q \in\left(\mathbf{L}^{\infty}\right)_{+}^{*}$, we may define

$$
\langle Q, X\rangle \triangleq \lim _{n} \uparrow\langle Q, X \wedge n\rangle \quad \in[0, \infty]
$$

for all $X \in \mathbf{L}_{+}^{0}$. For $X \in \mathbf{L}^{0}$, set $\langle Q, X\rangle=\left\langle Q, X^{+}\right\rangle-\left\langle Q, X^{-}\right\rangle$whenever this is well defined. Under this definition, it is easy to see that

$$
\langle Q, X\rangle \leq 0
$$

for all $Q \in \mathcal{D}$ and all $X \in \mathcal{C}_{0}$ which are uniformly bounded from below (actually, this holds for all $Q \in \mathcal{D}, X \in \mathcal{C}_{0}$ ).

We now define the value function of the dual optimization problem by

$$
v(y) \triangleq \min _{Q \in \mathcal{D}} J(y, Q):=\min _{Q \in \mathcal{D}}\left\{\mathrm{E}\left[V\left(y \frac{d Q^{r}}{d \mathbf{P}}\right)\right]+y\left\langle Q, e_{T}\right\rangle\right\},
$$

which is clearly decreasing and convex. The following is the principal result of the paper.

Theorem 3.1 Suppose Assumptions 1-3 hold true. Then

(i) $u(x)<\infty$ for all $x \in \mathrm{R}$ and $v(y)$ is finitely valued for all $y>0$. The value function $u$ and $v$ are conjugate in the sense that

$$
\begin{aligned}
& v(y)=\sup _{x>x_{0}}[u(x)-x y], \quad y>0, \\
& u(x)=\inf _{y>0}[v(y)+x y], \quad x>x_{0} .
\end{aligned}
$$


Here

$$
x_{0} \triangleq-v^{\prime}(\infty)=\sup _{Q \in \mathcal{D}}\left\langle Q,-e_{T}\right\rangle .
$$

The value function $u(x)$ is continuously differentiable on $\left(x_{0}, \infty\right)$ and $u(x)=-\infty$ for all $x<x_{0}$. The value function $v(y)$ is continuously differentiable on $(0, \infty)$.

(ii) For all $y>0$, there exists $\hat{Q}_{y} \in \mathcal{D}$ (unique up to the singular part) that attains the infimum in the dual problem (3.2). For all $x>x_{0}$, $\hat{X}=I\left(\hat{y} \frac{d \hat{Q}_{\hat{y}}^{r}}{d \mathbf{P}}\right)-x-e_{T}$ is optimal for the primal problem (2.1), where $\hat{y}$ attains the infimum of $[v(y)+x y]$, and $\hat{y}=u^{\prime}(x)$.

The proof of the above Theorem will be given in Section 4 below.

Remark 3.1 The definition of the dual domain $\mathcal{D}$ is independent of $x$ and $\left(e_{t}\right)_{0 \leq t \leq T}$. It is simply the polar set of $\mathcal{C}$ (intersected with the norm-1 elements). Moreover, $\mathcal{D}$ and $\mathcal{D}^{r}$ are both nonempty since $\mathcal{M} \subseteq \mathcal{D}^{r}$. Actually, $\mathcal{D}^{r}$ is the set of absolutely continuous local martingale measures in the case of locally bounded S (see DS[94], Theorem 5.6); see also DS[98] for the general case and the notion of equivalent sigma-martingale measures.

\section{Proof of the Main Theorem}

We claim that $\mathrm{E}\left[U\left(x+X+e_{T}\right)\right] \leq J(y, Q)+x y$ for any $X \in \mathcal{C}_{0}$ and $y>$ $0, Q \in \mathcal{D}$. We only need to consider the case $x+X+e_{T} \geq 0$ (hence $X$ is uniformly bounded from below). It follows from the definition of $V(\cdot)$, nonnegativity of $x+X+e_{T}$, and $\langle Q, X\rangle \leq 0$, that

$$
\begin{aligned}
\mathrm{E}\left[U\left(x+X+e_{T}\right)\right] & \leq \mathrm{E}\left[V\left(y \frac{d Q^{r}}{d \mathbf{P}}\right)+y\left(x+X+e_{T}\right) \frac{d Q^{r}}{d \mathbf{P}}\right] \\
& \leq \mathrm{E}\left[V\left(y \frac{d Q^{r}}{d \mathbf{P}}\right)\right]+y\left\langle Q, x+X+e_{T}\right\rangle \\
& \leq J(y, Q)+x y .
\end{aligned}
$$

Moreover, the above inequalities become equalities if and only if

$$
x+X+e_{T}=I\left(y \frac{d Q^{r}}{d \mathbf{P}}\right), \quad\left\langle Q^{s}, x+X+e_{T}\right\rangle=0 \quad \text { and } \quad\langle Q, X\rangle=0,
$$

in which case $X$ is optimal for the primal problem. It is now clear that

$$
u(x) \leq \inf _{y>0}[v(y)+x y]
$$


To show that equality actually holds true, it suffices to find a pair $(\hat{y}, \hat{Q})$ which attains the infimum of $[J(y, Q)+x y]$ and a $X \in \mathcal{C}_{0}$ such that equalities (4.6) hold.

First note that $v(y)$ is finitely valued. Indeed, it follows from Jensen's inequality and the decrease of $V(\cdot)$ that

$$
v(y) \geq \min _{Q \in \mathcal{D}} \mathrm{E}\left[V\left(y \frac{d Q^{r}}{d \mathbf{P}}\right)\right]-y \rho \geq \min _{Q \in \mathcal{D}} V\left(y \mathrm{E}\left[\frac{d Q^{r}}{d \mathbf{P}}\right]\right)-y \rho \geq V(y)-y \rho,
$$

where $\rho=\left\|e_{T}\right\|_{\infty}$. The fact $v(y)<\infty$ follows from Theorem 2.2(iv) KS[99] (observe $\mathcal{M} \subseteq \mathcal{D},\left\langle Q, e_{T}\right\rangle \leq \rho$ and $\sup _{X \in \mathcal{C}_{0}} E U(x+X) \leq u(x+\rho)<\infty$ for all $x)$.

The following inequalities are often used in the proof below. Under Assumption 2, there exist $y_{0}>0,0<\gamma, \mu<1$ and $C<\infty$ such that

$$
y I(y)<\frac{\gamma}{1-\gamma} V(y) \quad \text { and } \quad V(\mu y)<C V(y), \quad \forall 0<y<y_{0} .
$$

(see KS[99] Lemma 6.3 and Corollary 6.1 for the proof.)

Lemma 4.1 For every $y>0$, the minimum in the definition of the dual value function $v(y)$ is attained at some $\hat{Q}_{y} \in \mathcal{D}$.

Proof: With the help of Komlos Theorem (see Schwartz 86, for example) and convexity of $\mathcal{D}$, we can find a minimizing sequence $\left\{Q_{n}\right\} \subseteq \mathcal{D}$ such that

$$
\frac{d Q_{n}^{r}}{d \mathbf{P}} \longrightarrow f \quad \text { almost surely }
$$

for some random variable $f \geq 0$. Moreover, since $\left|\left\langle Q_{n}, e_{T}\right\rangle\right| \leq \rho$, we can always extract a subsequence of $Q_{n}$ (still denoted by $Q_{n}$ ) such that $\left\langle Q_{n}, e_{T}\right\rangle$ converges. Since $\mathcal{D}$ is weak-star closed and bounded, it is weak-star compact, and the sequence $\left\{Q_{n}\right\}$ has a cluster point $Q_{*} \in \mathcal{D}$ (that might not be unique). We want to show that $Q_{*}$ is actually a minimizer. It follows from Proposition A.1 that

By Lemma 3.4 of $\mathrm{KS}[99]$, we have

$$
\frac{d Q_{*}^{r}}{d \mathbf{P}}=f=\lim \frac{d Q_{n}^{r}}{d \mathbf{P}} .
$$

$$
\liminf \mathrm{E}\left[V\left(y \frac{d Q_{n}^{r}}{d \mathbf{P}}\right)\right] \geq \mathrm{E}\left[V\left(y \frac{d Q_{*}^{r}}{d \mathbf{P}}\right)\right] .
$$

Furthermore, since $\left\langle Q_{*}, e_{T}\right\rangle$ is a cluster point of $\left\{\left\langle Q_{n}, e_{T}\right\rangle\right\}$ which is convergent, we have $\left\langle Q_{*}, e_{T}\right\rangle=\lim \left\langle Q_{n}, e_{T}\right\rangle$. Hence $J\left(y, Q_{*}\right) \leq \liminf J\left(y, Q_{n}\right)=$ $v(y)$, which yields

$$
J\left(y, Q_{*}\right)=v(y) .
$$


Therefore, we can take $\hat{Q}_{y}=Q_{*}$.

Remark 4.1 The minimizer might NOT be unique. However, it is unique to the extent that its countably additive part (regular part) is unique. Indeed, suppose $Q_{1}, Q_{2}$ are two minimizers with $Q_{1}^{r} \neq Q_{2}^{r}$. Let $Q \triangleq \frac{1}{2} Q_{1}+\frac{1}{2} Q_{2}$. It follows that $Q^{r}=\frac{1}{2} Q_{1}^{r}+\frac{1}{2} Q_{2}^{r}$. By strict convexity of $V$ we have $\mathrm{E}\left[V\left(y \frac{d Q^{r}}{d \mathbf{P}}\right)\right]<$ $\frac{1}{2} \mathrm{E}\left[V\left(y \frac{d Q_{1}^{r}}{d \mathbf{P}}\right)\right]+\frac{1}{2} \mathrm{E}\left[V\left(y \frac{d Q_{2}^{r}}{d \mathbf{P}}\right)\right]$. Hence $J(y, Q)<\frac{1}{2} J\left(y, Q_{1}\right)+\frac{1}{2} J\left(y, Q_{2}\right)=$ $J\left(y, \hat{Q}_{y}\right)$, a contradiction.

Remark 4.2 It is easy to see that the function $v(\cdot)$ is actually strictly convex. Indeed, for all $y_{1}, y_{2}>0$ with $y_{1} \neq y_{2}$, and $\lambda \in(0,1)$, we have, for $y=\lambda y_{1}+(1-\lambda) y_{2}$,

$$
v(y)=J\left(y, \hat{Q}_{y}\right)>\lambda J\left(y_{1}, \hat{Q}_{y}\right)+(1-\lambda) J\left(y_{2}, \hat{Q}_{y}\right) \geq \lambda v\left(y_{1}\right)+(1-\lambda) v\left(y_{2}\right) .
$$

Lemma 4.2 The dual value function v(y) is continuously differentiable with

$$
v^{\prime}(y)=-\left\langle\hat{Q}_{y}^{r}, I\left(y \frac{d \hat{Q}_{y}^{r}}{d \mathbf{P}}\right)\right\rangle+\left\langle\hat{Q}_{y}, e_{T}\right\rangle .
$$

Proof: We first show that $v(\cdot)$ is differentiable (hence continuously differentiable by convexity). For a fixed $y>0$, let $h(z) \triangleq \mathrm{E}\left[V\left(z \frac{d \hat{Q}_{y}^{r}}{d \mathbf{P}}\right)\right]+z\left\langle\hat{Q}_{y}, e_{T}\right\rangle$. Then $h(\cdot)$ is convex, $h(\cdot) \geq v(\cdot)$ and $h(y)=v(y)$. These estimates easily imply $\triangle^{-} h(y) \leq \triangle^{-} v(y) \leq \triangle^{+} v(y) \leq{\Delta^{+}}^{+}(y)$, where ${\Delta^{ \pm}}^{ \pm}$denote the left and the right derivatives respectively. It is easy to show, by the fact that $V^{\prime}(\cdot)=-I(\cdot)$, and the Monotone Convergence Theorem, that

$$
\triangle^{+} h(y) \leq-\mathrm{E}\left[\frac{d \hat{Q}_{y}^{r}}{d \mathbf{P}} I\left(y \frac{d \hat{Q}_{y}^{r}}{d \mathbf{P}}\right)\right]+\left\langle\hat{Q}_{y}, e_{T}\right\rangle=-\left\langle\hat{Q}_{y}^{r}, I\left(y \frac{d \hat{Q}_{y}^{r}}{d \mathbf{P}}\right)\right\rangle+\left\langle\hat{Q}_{y}, e_{T}\right\rangle .
$$

On the other hand,

$$
\triangle^{-} h(y) \geq \limsup _{\epsilon \rightarrow 0+} \mathrm{E}\left[-\frac{d \hat{Q}_{y}^{r}}{d \mathbf{P}} I\left((y-\epsilon) \frac{d \hat{Q}_{y}^{r}}{d \mathbf{P}}\right)+\left\langle\hat{Q}_{y}, e_{T}\right\rangle\right] .
$$

We claim that, with $y_{0}$ being the constant from (4.9),

$$
\frac{d \hat{Q}_{y}^{r}}{d \mathbf{P}} I\left((y-\epsilon) \frac{d \hat{Q}_{y}^{r}}{d \mathbf{P}}\right)=\frac{d \hat{Q}_{y}^{r}}{d \mathbf{P}} I\left((y-\epsilon) \frac{d \hat{Q}_{y}^{r}}{d \mathbf{P}}\right) \mathbf{1}_{\left\{y \frac{\left.d \hat{Q}_{y}^{r} \leq y_{0}\right\}}{d \mathbf{P}}\right.}+\frac{d \hat{Q}_{y}^{r}}{d \mathbf{P}} I\left((y-\epsilon) \frac{d \hat{Q}_{y}^{r}}{d \mathbf{P}}\right) \mathbf{1}_{\left\{y \frac{d \hat{Q}_{y}^{r}}{d \mathbf{P}}>y_{0}\right\}}
$$


is uniformly integrable when $\epsilon$ is sufficiently small. Indeed, the second part is dominated by $I\left(\frac{y-\epsilon}{y} y_{0}\right) \frac{d \hat{Q}_{y}^{r}}{d \mathbf{P}}$, which is uniformly integrable when $\epsilon$ is small since $\left\|\hat{Q}_{y}^{r}\right\| \leq 1$. It follows from (4.9) that the first part, when $\epsilon$ is small, is dominated by

$$
\frac{1}{y-\epsilon} \frac{\gamma}{1-\gamma} V\left((y-\epsilon) \frac{d \hat{Q}_{y}^{r}}{d \mathbf{P}}\right)
$$

which is in turn dominated by

$$
\frac{1}{y-\epsilon} \frac{\gamma C}{1-\gamma} V\left(y \frac{d \hat{Q}_{y}^{r}}{d \mathbf{P}}\right)
$$

Therefore the first part is also uniformly integrable since $\mathrm{E}\left|V\left(y \frac{d \hat{Q}_{y}^{r}}{d \mathbf{P}}\right)\right|<\infty$. We have established

$$
\triangle^{-} h(y) \geq-\mathrm{E}\left[\frac{d \hat{Q}_{y}^{r}}{d \mathbf{P}} I\left(y \frac{d \hat{Q}_{y}^{r}}{d \mathbf{P}}\right)\right]+\left\langle\hat{Q}_{y}, e_{T}\right\rangle=-\left\langle\hat{Q}_{y}^{r}, I\left(y \frac{d \hat{Q}_{y}^{r}}{d \mathbf{P}}\right)\right\rangle+\left\langle\hat{Q}_{y}, e_{T}\right\rangle .
$$

This completes our proof.

Lemma $4.3 v^{\prime}(0+)=-\infty, v^{\prime}(\infty) \in\left[\inf _{Q \in \mathcal{D}}\left\langle Q, e_{T}\right\rangle, \sup _{Q \in \mathcal{D}}\left\langle Q, e_{T}\right\rangle\right]$

Proof: Observe that $v(0+) \geq V(0+)$ by (4.8). However, $v(y) \leq V(0+)+y \rho$ implies $v(0+) \leq V(0+)$, which in turn implies $v(0+)=V(0+)=U(\infty)$. If $U(\infty)=\infty$, then $v(0+)=\infty$ and $v^{\prime}(0+)=-\infty$. If $U(\infty)<\infty$, we have

$$
-v^{\prime}(0+) \geq \frac{v(0+)-v(y)}{y} \geq \frac{V(0+)-V\left(y \frac{d Q^{r}}{d \mathbf{P}}\right)-y \rho}{y} \geq \mathrm{E}\left[\frac{d Q^{r}}{d \mathbf{P}} I\left(y \frac{d Q^{r}}{d \mathbf{P}}\right)\right]-\rho
$$

for all $y>0$ and $Q \in \mathcal{D}$. Letting $y \rightarrow 0$, we have $-v^{\prime}(0+) \geq \infty$, or $v^{\prime}(0+)=-\infty$.

By de l'Hospital's Rule

$$
\begin{aligned}
v^{\prime}(\infty) & =\lim _{y \rightarrow \infty} \frac{v(y)}{y}=\lim _{y \rightarrow \infty} \frac{\inf _{Q \in \mathcal{D}}\left\{\mathrm{E}\left[V\left(y \frac{d Q^{r}}{d \mathbf{P}}\right)\right]+y\left\langle Q, e_{T}\right\rangle\right\}}{y} \\
& \in\left[\lim _{y \rightarrow \infty} \frac{\inf _{Q \in \mathcal{D}} \mathrm{E}\left[V\left(y \frac{d Q^{r}}{d \mathbf{P}}\right)\right]}{y}+\inf _{Q \in \mathcal{D}}\left\langle Q, e_{T}\right\rangle, \lim _{y \rightarrow \infty} \frac{\inf _{Q \in \mathcal{D}} \mathrm{E}\left[V\left(y \frac{d Q^{r}}{d \mathbf{P}}\right)\right]}{y}+\sup _{Q \in \mathcal{D}}\left\langle Q, e_{T}\right\rangle\right]
\end{aligned}
$$

However, $\lim _{y \rightarrow \infty} \frac{\inf _{Q \in \mathcal{D}} \mathrm{E}\left[V\left(y \frac{d Q^{r}}{d \mathbf{P}}\right)\right]}{y}=0$ as in KS[99], Lemma 3.7. 
Remark 4.3 Note that $\inf _{y>0}[v(y)+x y]=-\infty$ for all $x<-v^{\prime}(\infty)$, which implies $u(x)=-\infty$ by (4.7) (in this case the optimization problem is trivial). However, for every $x \in\left(-v^{\prime}(\infty), \infty\right)$, there exists a unique $\hat{y}>0$ that attains the infimum of $[v(y)+x y]$, and such that $v^{\prime}(\hat{y})=-x$. From now on we consider $x \in\left(-v^{\prime}(\infty), \infty\right)$, and let $\hat{Q}:=\hat{Q}_{\hat{y}}$ and $\hat{X}=I\left(\hat{y} \frac{d \hat{Q}^{r}}{d \mathbf{P}}\right)-x-e_{T}$. It follows from Lemma 4.2 that

$$
v^{\prime}(\hat{y})=-x=-\left\langle\hat{Q}^{r}, x+\hat{X}\right\rangle+\left\langle\hat{Q}^{s}, e_{T}\right\rangle .
$$

Lemma 4.4 We have

$$
\sup _{Q \in \mathcal{D}}\left[\left\langle Q^{r}, x+\hat{X}\right\rangle-\left\langle Q^{s}, e_{T}\right\rangle\right]=\left\langle\hat{Q}^{r}, x+\hat{X}\right\rangle-\left\langle\hat{Q}^{s}, e_{T}\right\rangle=x
$$

in particular,

$$
\left\langle Q^{r}, x+\hat{X}\right\rangle \leq\left\langle Q^{s}, e_{T}\right\rangle+x \quad \forall Q \in \mathcal{D} .
$$

Remark 4.4 ¿From the definition of $\mathcal{D}$, we have $\langle Q, x+\hat{X}\rangle \leq x$, for $Q \in \mathcal{D}$. If the endowment $e_{T}$ is zero almost surely, then, by the lemma, we also have $\left\langle Q^{r}, x+\hat{X}\right\rangle \leq x$ for $Q \in \mathcal{D}$, and $\left\langle\hat{Q}^{r}, x+\hat{X}\right\rangle=x$. This has the "classical" interpretation that $x$ is the cost of replicating the claim $\hat{A}_{T}:=x+\hat{X}$ in this incomplete market, and the "shadow state-price density" for pricing $\hat{A}_{T}$ is given by the density of $\hat{Q}^{r}$. In the case of a nonzero endowment process this interpretation is somewhat lost: now we have $\left\langle\hat{Q}, x+X_{T}\right\rangle=x$ (see below), but $\hat{Q}$ does not necessarily have a density.

For the agent receiving the endowment, the cost of financing $x+\hat{X}+e_{T}$ is still $x$, but

$$
\left\langle\hat{Q}^{r}, x+\hat{X}+e_{T}\right\rangle=x+\left\langle\hat{Q}, e_{T}\right\rangle .
$$

However, if the endowment process is "spanned" in the market, namely representable as $e_{t}=\left(H^{e} \cdot S\right)_{t}$ for some admissible strategy $H^{e}$, the standard interpretation is preserved. Indeed, since $e_{t}$ is assumed to be a bounded process, we have both $\left\langle Q, e_{T}\right\rangle \leq 0$ and $\left\langle Q,-e_{T}\right\rangle \leq 0$, for all $Q \in \mathcal{D}$. In particular, $\left\langle\hat{Q}, e_{T}\right\rangle=0$, and $x=\left\langle\hat{Q}^{r}, x+\hat{X}+e_{T}\right\rangle$.

Proof of Lemma 4.4: For a given $Q \in \mathcal{D}$ and $\epsilon \in(0,1)$, let $Q_{\epsilon} \triangleq(1-$ 
$\epsilon) \hat{Q}+\epsilon Q$. It follows that $Q_{\epsilon}^{r}=(1-\epsilon) \hat{Q}^{r}+\epsilon Q^{r}$. By optimality of $\hat{Q}$ we have

$$
\begin{aligned}
0 & \geq \frac{1}{\epsilon \hat{y}} \mathrm{E}\left[V\left(\hat{y} \frac{d \hat{Q}^{r}}{d \mathbf{P}}\right)-V\left(\hat{y} \frac{d Q_{\epsilon}^{r}}{d \mathbf{P}}\right)\right]+\left\langle\hat{Q}, e_{T}\right\rangle-\left\langle Q, e_{T}\right\rangle \\
& \geq-\frac{1}{\epsilon \hat{y}} \mathrm{E}\left[\hat{y}\left(\frac{d \hat{Q}^{r}}{d \mathbf{P}}-\frac{d Q_{\epsilon}^{r}}{d \mathbf{P}}\right) I\left(\hat{y} \frac{d Q_{\epsilon}^{r}}{d \mathbf{P}}\right)\right]+\left\langle\hat{Q}, e_{T}\right\rangle-\left\langle Q, e_{T}\right\rangle \\
& =\mathrm{E}\left[\left(\frac{d Q^{r}}{d \mathbf{P}}-\frac{d \hat{Q}^{r}}{d \mathbf{P}}\right) I\left(\hat{y} \frac{d Q_{\epsilon}^{r}}{d \mathbf{P}}\right)\right]+\left\langle\hat{Q}, e_{T}\right\rangle-\left\langle Q, e_{T}\right\rangle .
\end{aligned}
$$

However,

$$
\left(\left(\frac{d Q^{r}}{d \mathbf{P}}-\frac{d \hat{Q}^{r}}{d \mathbf{P}}\right) I\left(\hat{y} \frac{d Q_{\epsilon}^{r}}{d \mathbf{P}}\right)\right)^{-} \leq \frac{d \hat{Q}^{r}}{d \mathbf{P}} I\left(\hat{y} \frac{d Q_{\epsilon}^{r}}{d \mathbf{P}}\right) \leq \frac{d \hat{Q}^{r}}{d \mathbf{P}} I\left(\hat{y}(1-\epsilon) \frac{d \hat{Q}^{r}}{d \mathbf{P}}\right) .
$$

It follows from the same proof as in Lemma 4.2 that the last term is uniformly integrable when $\epsilon$ is sufficiently small. Now Fatou's Lemma gives

$$
\begin{aligned}
0 & \geq \mathrm{E}\left[\left(\frac{d Q^{r}}{d \mathbf{P}}-\frac{d \hat{Q}^{r}}{d \mathbf{P}}\right) I\left(\hat{y} \frac{d \hat{Q}^{r}}{d \mathbf{P}}\right)\right]+\left\langle\hat{Q}, e_{T}\right\rangle-\left\langle Q, e_{T}\right\rangle \\
& =\left\langle Q^{r}, x+\hat{X}\right\rangle-\left\langle\hat{Q}^{r}, x+\hat{X}\right\rangle+\left\langle\hat{Q}^{s}, e_{T}\right\rangle-\left\langle Q^{s}, e_{T}\right\rangle,
\end{aligned}
$$

which completes our proof.

We recall from DS[94] the following version of the bipolar theorem:

Lemma 4.5 Let $X \in \mathbf{L}^{\infty}$. Then $X \in \mathcal{C}$ if and only if $\langle Q, X\rangle \leq 0$ for all $Q \in \mathcal{D}^{r}$.

Proof: Necessity follows directly from the definition. For sufficiency, first note that the set $\mathcal{C}$ is a closed convex cone in $\mathbf{L}^{\infty}$ equipped with the weakstar topology $\sigma\left(\mathbf{L}^{\infty}, \mathbf{L}^{1}\right)$; see DS[94], Theorem 4.2 (for the case of non locally bounded $S$ we refer to DS[98]). Now let $X \in \mathbf{L}^{\infty}$, such that $\langle Q, X\rangle \leq 0$ for all $Q \in \mathcal{D}^{r}$. If $X$ does not belong to $\mathcal{C}$, by the Hahn-Banach Theorem $X$ and $\mathcal{C}$ are strictly separated (see Conway 85, Theorem IV.3.9.) Therefore, there exists a continuous linear functional $f$ (i.e. $\left.f \in\left(\mathbf{L}^{\infty}, \sigma\left(\mathbf{L}^{\infty}, \mathbf{L}^{1}\right)\right)^{*}=\mathbf{L}^{1}\right)$ such that $\langle f, X\rangle>\alpha$ and $\langle f, X\rangle \leq \alpha, \forall X \in \mathcal{C}$ for some $\alpha \in \mathbf{R}$. We claim that $\alpha=0$. Since $0 \in \mathcal{C}$, we have $\alpha \geq 0$. Moreover, if there exists an $X \in \mathcal{C}$ such that $\langle f, X\rangle>0$, then for any constant $c>0$, we have $c X \in \mathcal{C}$ because $\mathcal{C}$ is a convex cone. Thus, $\langle f, c X\rangle=c\langle f, X\rangle$ tends to $+\infty$ as $c \rightarrow \infty$, which 
is impossible. Hence $\alpha=0$. This implies that $f \in \mathcal{D}^{r}$ and $\langle f, X\rangle>0$, which is impossible.

Lemma 4.6 $\hat{X} \in \mathcal{C}_{0}$.

Proof: It suffices to show that $\hat{X} \wedge n \in \mathcal{C}$ for all $n>0$, and the rest follows from DS[94], Theorem 4.2 again. However, $\hat{X} \wedge n \in \mathbf{L}^{\infty}$ because $\hat{X}$ is uniformly bounded from below. Moreover, for any $Q \in \mathcal{D}^{r}$ we have $Q^{r}=Q$ and it follows from Lemma 4.4 that

$$
\langle Q, x+\hat{X} \wedge n\rangle \leq\langle Q, x+\hat{X}\rangle \leq x
$$

which implies $\langle Q, \hat{X} \wedge n\rangle \leq 0$ for all $Q \in \mathcal{D}^{r}$ and $n \geq 0$. By Lemma 4.5, we obtain $\hat{X} \wedge n \in \mathcal{C}$.

Remark 4.5 Since $\hat{X} \in \mathcal{C}_{0}$ and $\hat{X}$ is bounded from below, we have $\langle\hat{Q}, \hat{X}\rangle \leq 0$. However, it follows from (4.10) that

$$
\left\langle\hat{Q}, e_{T}\right\rangle+x=\left\langle\hat{Q}^{r}, x+\hat{X}+e_{T}\right\rangle \leq\left\langle\hat{Q}, x+\hat{X}+e_{T}\right\rangle \leq\left\langle\hat{Q}, e_{T}\right\rangle+x,
$$

which yields the last two equations in (4.6):

$$
\left\langle\hat{Q}^{s}, x+\hat{X}+e_{T}\right\rangle=0 \quad \text { and } \quad\langle\hat{Q}, \hat{X}\rangle=0 .
$$

Therefore, we have shown that $\hat{X}$ solves the primal optimization problem and

$$
u(x)=v(\hat{y})+x \hat{y} .
$$

Remark 4.6 By definition there exists an admissible portfolio process $\hat{H}$ such that $\hat{X}:=\hat{X}_{T}=(\hat{H} \cdot S)_{T}$. Let $\hat{X}_{t} \triangleq(\hat{H} \cdot S)_{t}$. We claim that $\hat{X}_{t}$ is a "martingale" under the finitely additive measure $\hat{Q}$ in the sense that

$$
\left\langle\hat{Q}, \hat{X}_{T} \mathbf{1}_{A}\right\rangle=\left\langle\hat{Q}, \hat{X}_{t} \mathbf{1}_{A}\right\rangle
$$

for all $A \in \mathcal{F}_{t}$. To this end, we only need to show that $\hat{X}_{t}$ is a "supermartingale" under the finitely additive measure $\hat{Q}$ ("martingale" property will follow from the fact that $\hat{X}_{0}=0$ and $\left\langle\hat{Q}, \hat{X}_{T}\right\rangle=0$ ), or equivalently

$$
\left\langle\hat{Q}, \hat{X}_{T} \mathbf{1}_{A}\right\rangle \leq\left\langle\hat{Q}, \hat{X}_{t} \mathbf{1}_{A}\right\rangle
$$


for all $A \in \mathcal{F}_{t}$. It suffices to show the above inequality for all $A \in \mathcal{F}_{t}$ on which $\hat{X}_{t}$ is bounded. Fix such a set $A$, and note that $\left(\hat{X}_{t}\right)$ is a supermartingale under any measure $Q \in \mathcal{D}^{r}$; see Proposition 4.7 DS[98]. This implies

$$
\left\langle Q, \hat{X}_{T} \mathbf{1}_{A} \wedge n\right\rangle \leq\left\langle Q, \hat{X}_{T} \mathbf{1}_{A}\right\rangle \leq\left\langle Q, \hat{X}_{t} \mathbf{1}_{A}\right\rangle, \quad \forall Q \in \mathcal{D}^{r}, \forall n>0
$$

Hence

$$
\left\langle\hat{Q}, \hat{X}_{T} \mathbf{1}_{A} \wedge n\right\rangle \leq\left\langle\hat{Q}, \hat{X}_{t} \mathbf{1}_{A}\right\rangle
$$

for all $n>0$, since $\mathcal{D}^{r}$ is weak-star dense in $\mathcal{D}$. Letting $n \rightarrow \infty$, we obtain

$$
\left\langle\hat{Q}, \hat{X}_{T} \mathbf{1}_{A}\right\rangle \leq\left\langle\hat{Q}, \hat{X}_{t} \mathbf{1}_{A}\right\rangle
$$

hence $\left(\hat{X}_{t}\right)$ is a "supermartingale" under the finite additive measure $\hat{Q}$, therefore also a "martingale".

Proof of the Main Theorem: The existence of the optimal $\hat{Q}_{y}$ for the dual problem, and the optimality of $\hat{X}$ for the primal problem have already been shown. We already know that $u(x) \leq v(y)+x y$, so that (4.13) implies (3.4). Then (3.3) is a consequence of the classical convex duality theory, as is the differentiability of $u$.

It only remains to show (3.5). From the argument above we obtain $|u(x)|<\infty$ for all $x>x_{0}$. This implies that there exists $X \in \mathcal{C}_{0}$ such that $x+X+e_{T} \geq 0$, hence $\left\langle Q, x+X+e_{T}\right\rangle \geq 0$, and $x \geq\left\langle Q,-e_{T}\right\rangle$, for all $Q \in \mathcal{D}$. It follows that $x_{0} \geq \sup _{Q \in \mathcal{D}}\left\langle Q,-e_{T}\right\rangle$, hence $x_{0}=\sup _{Q \in \mathcal{D}}\left\langle Q,-e_{T}\right\rangle$ from Lemma 4.3.

\section{Conclusions}

We characterize the optimal solution to the problem of maximizing utility from terminal wealth for an agent with random endowment, in general incomplete markets. This is done by appropriately defining the domain of the dual problem, as a subset of $\left(\mathbf{L}^{\infty}\right)^{*}$. As the referee points out, this result can be regarded as a necessary step towards the elusive general theory of equilibrium in incomplete semimartingale market models. Moreover, it can also serve as a stepping stone for the utility based approach to pricing contingent claims in incomplete markets, as in Hodges and Neuberger (1989). We leave these problems for future research. 


\section{A Appendix. Some properties of $\left(\mathbf{L}^{\infty}\right)_{+}^{*}$}

We state and prove here some well-known properties of $\left(\mathbf{L}^{\infty}\right)_{+}^{*}$, for the convenience of the reader. A more complete discussion can be found in Dunford and Schwartz (1967) (henceforth DS[67]) or Rao and Rao (1983).

Let $(\Omega, \mathcal{F}, \mathbf{P})$ be our underlying probability space and $\left(\mathbf{L}^{\infty}\right)^{*}$ be the dual space of $\mathbf{L}^{\infty}(\Omega, \mathcal{F}, \mathbf{P})$, and denote by $\left(\mathbf{L}^{\infty}\right)_{+}^{*}$ the set of all the nonnegative elements in $\left(\mathbf{L}^{\infty}\right)^{*}$. The set $\left(\mathbf{L}^{\infty}\right)_{+}^{*}$ can be identified as the set of all the nonnegative finitely additive bounded set functions on $\mathcal{F}$ which vanish on the sets of $\mathbf{P}$-measure zero (Theorem IV.8.16 of DS[67]). For any $Q \in\left(\mathbf{L}^{\infty}\right)_{+}^{*}$, there exists a unique decomposition

$$
Q=Q^{r}+Q^{s}, \quad Q^{r} \geq 0, \quad Q^{s} \geq 0,
$$

where $Q^{r}$ is countably additive (regular part) and $Q^{s}$ is purely finitely additive (singular part); see Definition III.7.7 and Theorem III.7.8 of DS[67] for relevant information. The measure $Q^{r}$ is absolutely continuous to $\mathbf{P}$, and we denote its Radon-Nikodym derivative $\frac{d Q^{r}}{d \mathbf{P}}$.

Lemma A.1 $Q \in\left(\mathbf{L}^{\infty}\right)_{+}^{*}$ is purely finitely additive (i.e. $Q^{r}=0$ ) if and only if for every $\epsilon>0$, there exists set $A_{\epsilon} \in \mathcal{F}$ such that $\mathbf{P}\left(A_{\epsilon}\right)>1-\epsilon$ and $\left\langle Q, 1_{A_{\epsilon}}\right\rangle=0$.

Proof: Sufficiency. Let $Q=Q^{r}+Q^{s}$ and $\frac{d Q^{r}}{d \mathbf{P}}=f$. Clearly $f=0$ on $A_{\epsilon}$ for any $\epsilon>0$. But $\mathbf{P}\left(A_{\epsilon}\right) \longrightarrow 1$, and we have $f=0$ almost surely, or $Q^{r}=0$.

Necessity. Suppose $Q^{r}=0$. We define the following new additive set function

$$
\nu(A) \triangleq \inf \left\{\left\langle Q, 1_{E}\right\rangle+\mathbf{P}(A \backslash E) ; \quad E \subseteq A, E \in \mathcal{F}\right\} ; \quad \forall A \in \mathcal{F} .
$$

It is fairly easy to show that $\nu$ is finitely additive; we omit the details. However, $\nu$ is actually countably additive (or, a measure) since $\nu\left(B_{n}\right) \leq$ $\mathbf{P}\left(B_{n}\right) \rightarrow 0$ whenever $\left\{B_{n} ; n \geq 1\right\}$ is a decreasing sequence of sets in $\mathcal{F}$ with $\cap_{n=1}^{\infty} B_{n}=\emptyset$. But $\nu \leq Q=Q^{s}$, which yields $\nu=0$ by definition.

Let $\epsilon>0$. Since $\nu(\Omega)=0$, there exists set $A_{n} \in \mathcal{F}$ for any $n>0$ such that

$$
\left\langle Q, 1_{A_{n}}\right\rangle<\frac{\epsilon}{2^{n}} \quad \text { and } \quad \mathbf{P}\left(A_{n}^{c}\right)<\frac{\epsilon}{2^{n}} .
$$

Let $A_{\epsilon} \triangleq \cap_{n=1}^{\infty} A_{n}$. Note $A_{\epsilon} \in \mathcal{F}$ and $\left\langle Q, 1_{A_{\epsilon}}\right\rangle \leq\left\langle Q, 1_{A_{n}}\right\rangle<\frac{\epsilon}{2^{n}}$, which implies that $\left\langle Q, 1_{A_{\epsilon}}\right\rangle=0$. On the other hand, $\mathbf{P}\left(A_{\epsilon}^{c}\right) \leq \sum_{n=1}^{\infty} \mathbf{P}\left(A_{n}^{c}\right)<\epsilon$, or $\mathbf{P}\left(A_{\epsilon}\right)>1-\epsilon$. This completes the proof. 
Proposition A.1 Suppose a sequence $\left\{Q_{n}\right\} \subseteq\left(\mathbf{L}^{\infty}\right)_{+}^{*}$ is such that $\frac{d Q_{n}^{r}}{d \mathbf{P}} \longrightarrow$ $f$ almost surely for some $f \geq 0$. Then any weak-star cluster point $Q$ of $\left\{Q_{n}\right\}$ satisfies $\frac{d Q^{r}}{d \mathbf{P}}=f$.

Proof: By Lemma A.1, for any $\epsilon>0$, there exists a set $A_{\epsilon} \in \mathcal{F}$ such that $\mathbf{P}\left(A_{\epsilon}\right)>1-\epsilon$ and $\left\langle Q^{s}, 1_{A_{\epsilon}}\right\rangle=0$. Moreover, there exist sets $B_{n} \in \mathcal{F}$ such that $\mathbf{P}\left(B_{n}\right)>1-\frac{\epsilon}{2^{n}}$ and $\left\langle Q_{n}^{s}, B_{n}\right\rangle=0$. By Egorov's Theorem, there exists a set $C_{\epsilon}$ such that $\mathbf{P}\left(C_{\epsilon}\right)>1-\epsilon$ and $\frac{d Q_{n}^{r}}{d \mathbf{P}} \longrightarrow f$ uniformly on $C_{\epsilon}$. Now for any $A \in \mathcal{F}$ such that $A \subseteq \Omega_{\epsilon} \triangleq A_{\epsilon} \bigcap_{n=1}^{\infty} B_{n} \cap C_{\epsilon}$, we have for a subsequence of $\left\{Q_{n}\right\}$ (still denoted as $\left\{Q_{n}\right\}$ )

$\int_{A} f d \mathbf{P}=\lim \int_{A} \frac{d Q_{n}^{r}}{d \mathbf{P}} d \mathbf{P}=\lim \left\langle Q_{n}^{r}, 1_{A}\right\rangle=\lim \left\langle Q_{n}, 1_{A}\right\rangle=\left\langle Q, 1_{A}\right\rangle=\left\langle Q^{r}, 1_{A}\right\rangle=\int_{A} \frac{d Q^{r}}{d \mathbf{P}} d \mathbf{P}$.

(The reason we can extract a subsequence is that $\left\langle Q, 1_{A}\right\rangle$ is a cluster point of $\left\langle Q_{n}, 1_{A}\right\rangle$.) Therefore, $\frac{d Q^{r}}{d \mathbf{P}}=f$ almost surely on $\Omega_{\epsilon}$, but $\mathbf{P}\left(\Omega_{\epsilon}\right)>1-3 \epsilon$. Letting $\epsilon \longrightarrow 0$, we complete the proof.

Corollary A.1 Let $Q_{n}$ be a sequence of purely finitely additive set functions with $Q$ as a weak-star cluster point. Then $Q$ is purely finitely additive.

\section{References}

[1] BISMUT, J.M. (1973) Conjugate convex functions in optimal stochastic control. J. Math. Anal. Appl. 44, 384-404.

[2] CONWAY, J.B. (1985) A Course in Functional Analysis. Springer, New York.

[3] CUOCO, D. (1997) Optimal consumption and equilibrium prices with portfolio constraints and stochastic income. J. Econ. Theory $\mathbf{7 2 .}$

[4] DELBAEN, F. \& SCHACHERMAYER, W. (1994) A general version of the fundamental theorem of asset pricing. Math. Annalen 300, 463-520.

[5] DELBAEN, F. \& SCHACHERMAYER, W. (1998) The fundamental theorem of asset pricing for unbounded stochastic processes. Math. Annalen 312, 215-250.

[6] DUfFie, D., FLEMING, V., SONER, M. \& ZARIPHOPOULOU (1997) Hedging in incomplete markets with HARA utility. J. Econ. Dynamics and Control 21, 753-782. 
[7] DUNFORD, N. \& SCHWARTZ, J.T. (1967) Linear Operators I. John Wiley \& Sons.

[8] HODGES, S.D. \& NEUBERGER, A. (1989) Optimal replication of contingent claims under transaction costs. Review of futures markets $\mathbf{8}$, 222-239.

[9] EL KAROUI, N. \& JEANBLANC, M. (1998) Optimization of consumption with labor income. Finance \& Stochastics 4, 409-440.

[10] KARATZAS, I., LEHOCZKY, J. P., ShreVE, S. E. \& XU, G. L. (1991) Martingale and duality methods for utility maximization in an incomplete market. SIAM J. Control Optimization 29, 702-730.

[11] KRAMKOV, D. \& SCHACHERMAYER, W. (1999) The asymptotic elasticity of utility functions and optimal investment in incomplete markets. To appear in The Annals of Applied Probability.

[12] PLISKA, S.R. (1986) A stochastic calculus model of continuous trading: optimal portfolio. Math. Oper. Res. 11, 371-382.

[13] RAO, B. \& RAO, B. (1983) Theory of Charges. Academic Press, New York.

[14] SCHWARTZ, M. (1986) New proofs of a theorem of Komlós. Acta Math. Hung. 47, 181-185. 